OPEN ACCESS

Edited by:

Xiaotong Wang,

Ludong University, China

Reviewed by:

Nelly Tremblay,

Fisheries and Oceans Canada,

Canada

Lorenzo Gallus

University of Genoa, Italy

Chenghua Li,

Ningbo University, China

*Correspondence.

Libin Zhang

zhanglibin@qdio.ac.cn

Specialty section:

This article was submitted to

Aquatic Physiology

a section of the journal

Frontiers in Marine Science

Received: 28 July 2021

Accepted: 04 October 2021

Published: 22 October 2021

Citation:

Ru X, Zhang $L$ and Yang $H$ (2021)

Plasticity of Locomotor Activity

Permits Energy Homeostasis During

Reproduction in a Female Sea

Cucumber. Front. Mar. Sci. 8:748571.

doi: 10.3389/fmars.2021.748571

\section{Plasticity of Locomotor Activity Permits Energy Homeostasis During Reproduction in a Female Sea Cucumber}

\author{
Xiaoshang $\mathrm{Ru}^{1,2,3,4,5}$, Libin Zhang ${ }^{1,2,3,4,5 *}$ and Hongsheng Yang ${ }^{1,2,3,4,5,6}$ \\ ${ }^{1}$ CAS Key Laboratory of Marine Ecology and Environmental Sciences, Institute of Oceanology, Chinese Academy \\ of Sciences, Qingdao, China, ${ }^{2}$ Laboratory for Marine Ecology and Environmental Science, Qingdao National Laboratory \\ for Marine Science and Technology, Qingdao, China, ${ }^{3}$ Center for Ocean Mega-Sciences, Chinese Academy of Sciences, \\ Qingdao, China, ${ }^{4}$ CAS Engineering Laboratory for Marine Ranching, Institute of Oceanology, Chinese Academy of Sciences, \\ Qingdao, China, ${ }^{5}$ Shandong Province Key Laboratory of Experimental Marine Biology, Qingdao, China, ${ }^{6}$ The Innovation \\ of Seed Design, Chinese Academy of Sciences, Wuhan, China
}

Behavioral plasticity in animals allows for moment-by-moment behavioral adjustments to biotic and abiotic uncertainties in the environment. For most aquatic animals, reproduction involves gonadal development and other physiological changes, causing increasing demands in nutrients and energy for females. The aim of this report was to determine how the female sea cucumber, Apostichopus japonicus, accommodates increasing energy demands during reproduction by adopting a behavioral energy conservation strategy. Dynamic changes in feeding activity, total body mass, locomotor activity, energetic condition, and metabolic performance of the females were measured from the non-breeding stage up to the mature stage. Routine metabolic rate analysis showed that reproduction caused a significant increase in energy demands in the adult. However, reproduction also suppressed the appetite of adults resulting in decreased energy intake. Interestingly, combining time-lapse camera and behavioral analysis software, the results showed that sea cucumbers down-regulated their locomotor activities in order to conserve energy effectively. Energy budget analysis and unchanged cortisol level revealed that the reduction in motility offset the increased energy demand for reproduction while helping to maintain energy homeostasis in the maternal body. Altogether, our study suggests that plasticity in the locomotor behavior allows sea cucumbers to cope with the high energy demands of reproduction.

Keywords: sea cucumber, behavioral plasticity, energetic physiology, energy homeostasis, reproduction

\section{INTRODUCTION}

Reproduction is one of the most important events of all organisms. However, reproduction is energetically costly (Speakman, 2008). For example, gonadal development, metabolic maintenance, behavioral thermoregulation, and brood care require the expenditure of considerable amounts of energy in many aquatic animals (Baeza and Fernández, 2002; Fossette et al., 2012; McBride et al., 2015; Duisan et al., 2021). As a result, activities associated with reproduction pose energetic challenges, particularly to females. 
Maintaining energy homeostasis is critical for development, growth, reproduction, and survival (Unniappan et al., 2020). Interestingly, as a response to variations in energy or nutrient demands at different life stages (i.e., reproduction), animals have evolved the capacity to adjust their behavioral and physiological functions in order to accommodate changing energy demands (Nie et al., 2015). For example, plasticity in physiological adjustment involves remodeling gut structure, and adjusting digestive strategy to increase digestive and absorptive efficiency (Krockenberger and Hume, 2007; Reiff et al., 2015). In addition, behavioral adjustments may involve expanding the home range, increasing foraging time, and shifting prey choice to increase energy intake (Barclay, 1989; Rodríguez et al., 2010; Sokolov et al., 2014).

Locomotor ability is a crucial necessity for survival. However, the development of eggs or fetuses places an additional physical burden on females (McLean and Speakman, 2000; Miles et al., 2000; Pruitt and Troupe, 2010). As a result, loss of or decrease in locomotor performance has been widely reported in terrestrial animals over the past several decades. Due to buoyancy, growing eggs may have no effect on locomotor ability in aquatic animals, especially in marine invertebrates that have a large body surface area. However, decreased locomotor activity under certain circumstances has been observed in many aquatic organisms during reproduction, including fish, echinoderms, aquatic snakes, and sea turtles (James and Johnston, 1998; Winne and Hopkins, 2006; Fossette et al., 2012; Ru et al., 2017). Recently, the behavioral energy conservation hypothesis has been proposed in order to explain unexpected behavior in aquatic animals (Fossette et al., 2012). This hypothesis states that plasticity of locomotor behavior allows animals to down-regulate their motility in order to conserve energy for other functions (such as reproduction and growth), thereby maximizing their fitness (Fossette et al., 2012; Okuyama et al., 2013). However, there is little physiological evidence to support this hypothesis.

In the present study, the sea cucumber, Apostichopus japonicus, was used as model system to test the behavioral energy conservation hypothesis during reproduction. Recent studies have reported that $A$. japonicus shows a series of behavioral and physiological changes after the onset of the annual reproductive cycle (Ru et al., 2017, 2021). Since both locomotion and egg production are high energy demand functions, we hypothesized that decreasing locomotor activity can compensate for the increased energy demand of reproduction. The behavioral energy conservation strategy might also have additional benefits for breeding females, such as maintaining maternal energy homeostasis. However, the relationship between reproduction, locomotor behavior, and energetic physiology remains unclear and requires further testing.

To elucidate this point, we analyzed the dynamic interaction of feeding activity, locomotor activity, metabolic performance, growth function, gonadal development, and energetic condition in female sea cucumber during reproduction. We also used a mechanistic model to show how plasticity of locomotor function maintains energy homeostasis during reproduction. Our study provides new insights into the interaction of locomotor behavior and energetic physiology during reproduction in sea cucumber.

\section{MATERIALS AND METHODS}

\section{Animals and Rearing Conditions}

In this study, sea cucumbers were collected in May 2017 from Rushan Bay, China $\left(36^{\circ} 46^{\prime} \mathrm{N}, 121^{\circ} 34^{\prime} \mathrm{E}\right)$. Animals were induced to spawn using the air-drying method (Liu et al., 2015). Only females were used in this study, since feeding activity, immune function, and locomotor activity of females may be affected by the pheromones of males (Jiang et al., 2017, 2020; Marquet et al., 2018). Identification of females was conducted by observation of oocyte release during spawning. After spawning, the females were identified, selected, and reared in three $5 \mathrm{~m} \times 3 \mathrm{~m} \times 2 \mathrm{~m}$ cages in an open-air pond located in the National Algae and Sea Cucumber Engineering Technique Research Center of China until November. Prior to the start of the annual reproductive cycle, 34 females $(126.93 \pm 5.02 \mathrm{~g})$ were collected randomly as the experimental animals.

The experiment lasted for 100 days. Prior to the study, 10 sea cucumbers were sampled as the non-breeding group. During the study, procedures were conducted every 25 days as follows: (1) All individuals $(n=24)$ were weighted to assess growth dynamics. (2) Six adults were randomly selected for behavioral activity recordings. (3) Six adults were randomly selected to measure metabolic performance.

During the study, sea cucumbers were reared in four $350 \mathrm{~L}$ plastic aquaria, with the stocking density of six individuals per tank. The animals were fed to satiation at 18:00. Feces were siphoned from each tank at 8:00 am daily, and then frozen at $-20^{\circ} \mathrm{C}$ for future analysis. Additional conditions: water temperature was $14^{\circ} \mathrm{C}$ controlled by a running water system, $\mathrm{pH}$ was $\sim 7.9$, dissolved oxygen concentration was $8.0 \mathrm{mg} \mathrm{L}^{-1}$, and the light intensity was 25 lux.

\section{Locomotor Activity Analysis}

At each stage, six females were randomly selected and individually placed in a white plastic cylinder $(50 \mathrm{~cm}$ in diameter) for $24 \mathrm{~h}$. Movement of each animal was recorded by a TLC 200 Brinno time-lapse camera. Environmental factors have a significant effect on the behavioral performance of sea cucumbers (Pan et al., 2015; Sun et al., 2018). Therefore, water temperature and light intensity in the cylinders were kept the same as rearing tanks throughout the recording.

Videos were saved as AVI files of $680 \times 480$ pixels and analyzed by XT Ethovison 10.1 software (Noldus Information Technology, Wageningen, Netherlands). The color difference between the animal and the background was used to catch and trace the movement of the sea cucumber during the behavioral analysis. The total locomotor distance in $24 \mathrm{~h}$ and total locomotor time in $24 \mathrm{~h}$ were used as functional parameters to assess the locomotor activity of sea cucumbers during the experiment.

\section{Measurement of Metabolic Performance}

The routine metabolic rate (RMR) of sea cucumbers was measured using an established method (Dong and Dong, 2006). Six sea cucumbers were randomly selected and each individual was placed in a $2.8 \mathrm{~L}$ sealed respiratory chamber (Subo Co., 
Ltd., Chongzhou, China) with the same environmental factors as the rearing tanks. After $2 \mathrm{~h}$, the oxygen consumption rate of each animal was determined using the ECO Sense ODO200 Do meter (YSI Co., Ltd., Yellow Springs, OH, United States). Four blank controls were measured to correct for the oxygen consumption of microorganisms in water. To minimize the effects of circadian rhythm and light condition on metabolic performance, all tests were carried out at 00:00. Therefore, RMR was calculated following the equation (Dong and Dong, 2006):

$$
\operatorname{RMR}\left(\mu \mathrm{gO}_{2} \mathrm{~g}^{-1} \mathrm{~h}^{-1}\right)=1000\left(\mathrm{D}_{t}-\mathrm{D}_{0}\right) \mathrm{V} / \mathrm{WT}
$$

where $\mathrm{D}_{\mathrm{t}}$ is the change in oxygen level $\left(\mathrm{mg} \mathrm{O}_{2} \mathrm{~L}^{-1}\right)$ in the test respiratory chamber with animal, $\mathrm{D}_{0}$ is the change in oxygen level $\left(\mathrm{mg} \mathrm{O}_{2} \mathrm{~L}^{-1}\right)$ in the test respiratory chamber without animal, $\mathrm{V}$ is the volume of the respiratory chamber $(\mathrm{L}), \mathrm{W}$ is the total body weight of the test sea cucumber $(\mathrm{g}), \mathrm{T}$ is the test time (h).

\section{Feeding Activity}

Daily food consumption (FC) and ingestion rate (IR) were used to assess the feeding activity of females during reproduction. FC and IR were determined using acidic insoluble ash markers (AIA, a natural indicator of food intake) in feces and diet according to an established protocol ( $\mathrm{Ru}$ et al., 2018). A $1 \mathrm{~g}$ sample was carbonized using a muffle furnace for $2 \mathrm{~h}$ at $300^{\circ} \mathrm{C}$ and $15 \mathrm{~h}$ at $600^{\circ} \mathrm{C}$ to remove organic matter. The ash was acidized, filtered, and then burned again at $600^{\circ} \mathrm{C}$ for $15 \mathrm{~h}$ to obtain the AIA value.

\section{Sample Collection and Determination of Cortisol Level}

All individuals were euthanized by decollation on day 100 . The gonad, body wall, respiratory tree, and gut of each animal were separated immediately under sterile conditions. In addition, a total of 10 individuals were randomly selected for sample collection (i.e., two, three, three, and two individuals were sampled from tank $1, \tan k 2$, tank3, and tank4, respectively). $2 \mathrm{~mL}$ of coelomic fluid from each individual was removed with a sterile syringe for cortisol determination, and the whole gonad and somatic tissues (i.e., body wall, gut and respiratory tree) of each individual were weighted and frozen at $-20^{\circ} \mathrm{C}$ for measurement of energy density.

Cortisol levels were determined by the enzyme linked immunosorbent (ELISA) assay method. We purchased the commercial ELISA Assay Kit from Nanjing Jiancheng Bioengineering Institute (Nanjing, China), and the determination was performed under the manufacturer's guidelines. The ELISA Assay Kit has been validated in many previous studies (Xu et al., 2020).

In details, prior to assay, coelomic fluid was clarified by centrifugation at $2,000 \mathrm{~g}$ for $10 \mathrm{~min}$ at $4^{\circ} \mathrm{C}$. And then $40 \mu \mathrm{L}$ supernatant of each sample was mixed with $10 \mu \mathrm{L}$ cortisol antibody and $50 \mu \mathrm{L}$ streptavidin-HRP in the test well. After the test well was sealed, the processed sample was incubated for $60 \mathrm{~min}$ in a water bath at $37^{\circ} \mathrm{C}$. After the procedures of washing and chromogen for $10 \mathrm{~min}$, the color development was terminated by stop solution. Finally, the cortisol level was determined at $450 \mathrm{~nm}$ using a DG5033A microplate reader (Huadong Electronics Group, Nanjing, China).

\section{Energy Budget}

Energy density of all samples, including somatic tissues, gonadal tissues, diet and feces, were measured using a IKA C600 calorimeter (IKA ${ }^{\circledR}$-Werke GmbH \& Co., KG, Staufen, Germany).

The daily activities of sea cucumbers are very simple, they move randomly to search food at night, and rest under a shelter during the day. A. japonicus is an income breeder (Ru et al., 2021). With the exception of egg development, energy from ingested food during reproduction also is invested into physiological metabolism, locomotor function, and growth function. Most unused nutrients were discharged from the body as feces (Yuan et al., 2007).

Therefore, an energy budget equation was constructed to investigate how sea cucumbers adjust their locomotor function to maintain energy homeostasis during reproduction as follows (Yuan et al., 2007, 2016):

$$
\mathrm{C}=\mathrm{F}+\mathrm{R}+\mathrm{B}+\mathrm{P}+\mathrm{G}
$$

where $C\left(\mathrm{~J}_{\text {ind }}^{-1} \mathrm{day}^{-1}\right)$ is the energy intake from ingested resources during reproduction, $\mathrm{F}\left(\mathrm{J}_{\text {ind }}{ }^{-1}\right.$ day $\left.^{-1}\right)$ is discharged energy as feces, $\mathrm{R}$ ( $\mathrm{J}$ ind ${ }^{-1} \mathrm{day}^{-1}$ ) is energy allocated into reproduction (i.e., gonadal growth), $\mathrm{P}\left(\mathrm{J}_{\mathrm{ind}}{ }^{-1} \mathrm{day}^{-1}\right)$ is energy lost in maintaining metabolic function, $\mathrm{B}\left(\mathrm{J} \mathrm{ind}^{-1} \mathrm{day}^{-1}\right)$ is energy used in locomotor function, and $G\left(\mathrm{~J}_{\text {ind }}{ }^{-1}\right.$ day $\left.^{-1}\right)$ is the deposited energy as somatic reserves. The six parameters were calculated as follows:

$$
\mathrm{C}=\mathrm{FC} \times \mathrm{E}_{\text {diet }} ; \mathrm{F}=\mathrm{FP} \times \mathrm{E}_{\text {feces }}
$$

where $E_{\text {diet }}$ and $E_{\text {feces }}$ are the energy density of diet and feces, FC is the daily food consumption, and FP is the daily feces production.

$$
\mathrm{R}=\Delta \mathrm{W}_{\mathrm{g}} \times \mathrm{E}_{\text {gonad }} ; \mathrm{G}=\Delta \mathrm{W}_{\mathrm{s}} \times \mathrm{E}_{\text {soma }}
$$

where $\mathrm{E}_{\text {gonad }}$ and $\mathrm{E}_{\text {soma }}$ are the energy density of gonadal tissues and somatic tissues, and $\Delta \mathrm{W}_{\mathrm{g}}$ and $\Delta \mathrm{W}_{\mathrm{s}}$ are the increased weight of gonadal and somatic tissues, respectively. The growth rate of somatic tissues and gonadal tissues of sea cucumbers at different breeding stages (every 25 days over 100 days) were 48.81, 28.53, $13.4,9.27 \%$ and $7.53,23.66,33.79$, and $35.02 \%$, respectively (Ru et al., 2018).

Energy used to maintain routine metabolic function was evaluated by nitrogen excretion. Sea cucumbers use 24,830 J of energy to excrete $1 \mathrm{~g}$ nitrogen (Yuan et al., 2016). The ratio of oxygen consumption to nitrogen excretion is 1:15 (Yuan et al., 2006). Therefore, $P$ was calculated following the equation:

$$
\mathrm{P}=\mathrm{RMR} \times 15 \times 24830 \times 24
$$

Lastly, the value of B was calculated by:

$$
\mathrm{B}=\mathrm{C}-\mathrm{F}-\mathrm{R}-\mathrm{P}-\mathrm{G}
$$




\section{Statistical Analysis}

Sea cucumbers show great individual variations in growth and physiological condition (Liang et al., 2010). Therefore, total body mass, body wall mass, gonad mass, metabolic rate, and locomotor activity were analyzed at the individual level. It is not possible to distinguish each cucumber for food ingestion and feces production in a same tank. Therefore, feeding activity and parameters in energy budget equation were analyzed between different tanks at different breeding stages. In order to avoid the pseudoreplication for the final analysis, data of cortisol level and energy density from the same tank were averaged and treated as one replicate.

All data were examined for normality distribution and homogeneity of variance by the Kolmogorov-Smirnov test and the Levene's test. Differences in body wall mass, gonad mass and cortisol levels at the non-breeding stage and the mature stage were analyzed by Student's $t$-test.

For normal data, changes in feeding activity, locomotor time, and parameters in the energy allocation model over time were analyzed by one-way ANOVA followed by Tukey's post hoc comparisons. For data with unequal variances, total body mass, $\mathrm{R}$, and locomotor distance were analyzed by the Kruskal-Wallis test.

Body size has a significant effect on physiological traits in marine organisms. Thus, the correlation between body weight and routine metabolic rate was analyzed using the Pearson correlation analysis among all individuals. The routine metabolic rate at different breeding stages were also analyzed by one-way analysis of covariance with the least significant difference test with changing body mass as a covariate.

\section{RESULTS}

\section{Body Growth and Gonad Development}

The total body mass of $A$. japonicus showed a continuous increase during reproduction (Figure 1A; Kruskal-Wallis test: $\left.H_{4}=71.557, P<0.001\right)$. The gonad mass reached $37.90 \mathrm{~g}$ over 100 days (Figure 1C; independent-samples $t$-test: $t_{32}=-19.301$, $P<0.001)$, suggesting that gonads were in the mature stage. It was found that the body wall mass at the mature stage was also significantly higher than that at the non-breeding stage (Figure 1B; $t_{32}=-13.007, P<0.001$ ).

\section{Feeding Activity}

Feeding activity, including daily food consumption rate and ingestion rate, is shown in Table 1 . Both daily food consumption (one-way ANOVA: $F_{3,12}=40.111, P<0.001$ ) and ingestion rate $\left(F_{3,12}=63.033, P<0.001\right)$ decreased significantly during reproduction.

\section{Routine Metabolic Rate and Cortisol Levels}

Pearson correlation analysis indicated that the routine metabolic rate was positively correlated with the total body mass (Figure 2A; $r=0.568, P<0.001$ ). As expected, the routine metabolic rate gradually increased during reproduction
(ANCOVA: $F_{4,25}=8.261, P<0.001$ ). However, the cortisol levels did not differ significantly between the non-breeding stage and the mature stage (Figure 2B; $t=-0.319, d f=12, P=0.755$ ).

\section{Locomotor Activity}

Dynamic change in locomotor activity of sea cucumbers during reproduction was assessed by the total locomotor distance and the total locomotor time in $24 \mathrm{~h}$. However, both locomotor distance (Figure 3A; Kruskal-Wallis test: $H_{4}=22.400, P<0.001$ ) and locomotor time (Figure 3B; oneway ANOVA: $\left.F_{4,25}=31.726, P<0.001\right)$ showed continuous decline during reproduction.

\section{Energy Intake and Allocation Patterns}

Energy acquisition in females ranged from $11,253.74$ to $6,656.89 \mathrm{~J}$ ind $^{-1}$ day $^{-1}$ during reproduction, showing a continuous downward trend (Figure 4A; one-way ANOVA: $F_{3,12}=40.111$, $P<0.001)$. Increasing energy was discharged from the body as feces over gonadal growth (Figure 4B; $F_{3,12}=18.536, P<0.001$ ). Energy allocated to metabolic functions increased significantly during reproduction (Figure 4B; $F_{3,12}=56.767, P<0.001$ ). Energy allocated to reproduction increased significantly at different breeding stages (Figure 4B; Kruskal-Wallis test: $\left.H_{3}=13.257, P=0.004\right)$. Energy allocated to locomotor function and somatic growth decreased significantly over time (Figure 4B; $\left.F_{3,12}=8.307, P=0.003 ; F_{3,12}=32.663, P<0.001\right)$.

\section{DISCUSSION}

\section{Increasing Energy Demand and Decreased Appetite During Reproduction}

Metabolic rate is a key indicator reflecting the state of energy expenditure in aquatic organisms (Huang et al., 2020; Pang et al., 2020). Compared to the non-breeding stage, the increase in sea cucumber metabolic rates during the breeding stage indicate that reproduction induces an increase in energy demand. Sea cucumbers, as ectotherms, are sensitive to both abiotic and biotic challenges. For example, variations in ambient temperature lead to an increase in metabolic rate (Dong et al., 2006). In addition, internal states, such as starvation and estivation, lead to a decrease in metabolic rate (Bao et al., 2010). In our study, adults were reared in suitable and stable conditions. Therefore, the growing gonad is the key factor inducing the increased metabolic rate in sea cucumbers. Similar results were also reported in fish, shellfish, and crustaceans (Boehlert et al., 1991; Baeza and Fernández, 2002; Kraffe et al., 2008). There are two reasons for increasing energy expenditure during reproduction. First, active egg growth requires high amounts of energy directly (from 228.83 to $1,063.72 \mathrm{~J} \mathrm{day}^{-1}$ at different breeding stages), and second the excretion of metabolic wastes (i.e., ammonia) from egg development through the maternal circulation also consumes energy indirectly (from 121.11 to $242.14 \mathrm{~J} \mathrm{day}^{-1}$ at different breeding stages). 

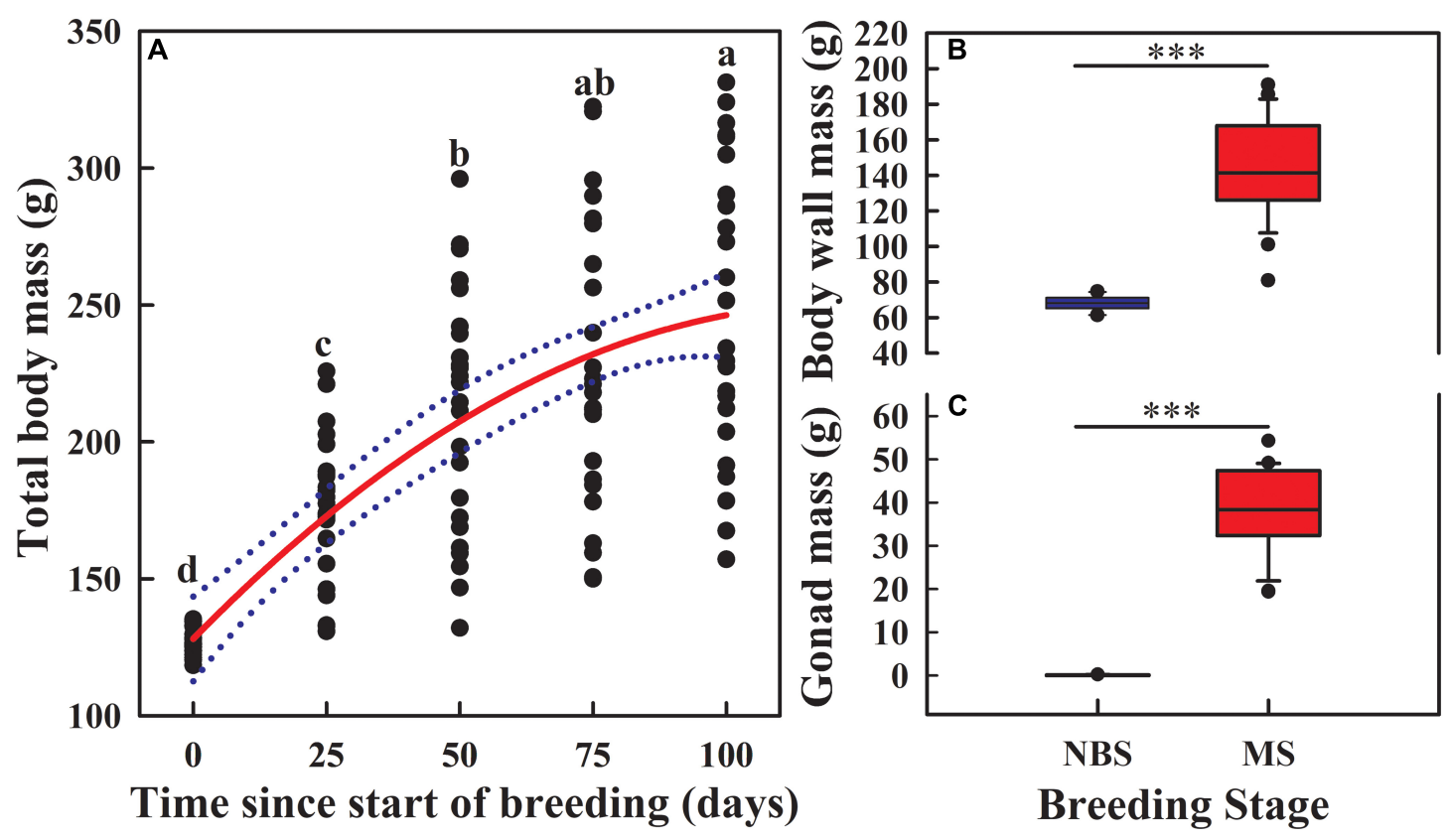

FIGURE 1 | Dynamic changes in total body mass (A), body wall mass (B) and gonad mass (C) of sea cucumbers during reproduction. Different letters indicate significant differences on different days $(P<0.05)$. Dotted lines represent $95 \%$ confidence intervals. Asterisk indicates a significant difference between the non-breeding stage (NBS) and the mature stage (MS). ${ }^{* * *} P<0.001$.

Sea cucumbers feed on sediments (Zhang et al., 2016). When food is easy to obtain, breeding animals may up-regulate their feeding activity to accommodate the increasing energy demand (Xu et al., 2012). Conversely, although females were provided a sufficient diet, their feeding activity showed a downward trend over gonad growth without interference from the courtship behavior of males (Marquet et al., 2018; Ru et al., 2018). This unexpected result suggests that reproduction suppresses the sea cucumber appetite. The contradictory phenomenon between increasing energy demand and suppressed feeding behavior has been widely reported for marine animals. Interestingly, many species such as octopus (Amphioctopus fangsiao), leatherback turtle (Dermochelys coriacea), and Atlantic salmon (Salmo salar) cease feeding completely during reproduction (Kadri et al., 1995; Plot et al., 2013; Tziouveli and Yokoyama, 2017). In our study, potential reasons for the loss of appetite in breeding females were not explored.

TABLE 1 | Dynamic changes in feeding activity of sea cucumbers during reproduction.

Time since start of breeding (days)

\begin{tabular}{|c|c|c|c|c|}
\hline Feeding activity & Days 0-25 & Days 25-50 & Days 50-75 & Days $75-100$ \\
\hline $\begin{array}{l}\text { Food consumption } \\
\text { (g ind }^{-1} \text { day }^{-1} \text { ) }\end{array}$ & $3.67 \pm 0.26^{a}$ & $3.18 \pm 0.18^{b}$ & $2.49 \pm 0.26^{c}$ & $2.17 \pm 0.12^{\mathrm{c}}$ \\
\hline $\begin{array}{l}\text { Ingestion rate } \\
\left(\mathrm{mg} \mathrm{g}^{-1} \text { day }^{-1}\right)\end{array}$ & $24.46 \pm 2.55^{a}$ & $16.57 \pm 1.43^{b}$ & $11.41 \pm 1.45^{\mathrm{c}}$ & $9.18 \pm 1.01^{\mathrm{C}}$ \\
\hline
\end{tabular}

Data are represented as mean and standard deviation of the mean.

Different letters in the same row represent a significant difference $(P<0.05)$.
However, we found that there were no changes in cortisol levels between the non-breeding and breeding stages in sea cucumbers. Cortisol level is associated with the endocrine response to energy homeostasis in sea cucumbers (Pei et al., 2012). The unchanged cortisol levels during reproduction suggests that the increased energy demand does not cause a negative effect on maternal energy homeostasis, even though sea cucumbers lose their appetite.

\section{Plasticity of Locomotor Function and Energy Homeostasis During Reproduction}

Loss of locomotor ability during reproduction has been widely considered a cost of reproduction (Seigel et al., 1987). The physical burden hypothesis has been proposed to explain these observations (Miles et al., 2000; Shine, 2003). This hypothesis suggests that the growing egg or embryo presents an additional burden for females. Therefore, reproductive status causes inevitable inhibition of locomotor function. However, sea cucumbers have a fluid-filled body cavity, due to buoyancy, the growing eggs do not add to the physical burden of breeding sea cucumbers (Hamel et al., 2019). Furthermore, in order to avoid potential interference on locomotor activity from courtship behavior and thermoregulation (Fossette et al., 2012; Ru et al., 2017; Marquet et al., 2018), all individuals used in this study were females and the ambient temperature was maintained at $14^{\circ} \mathrm{C}$. However, the locomotor activity of females still decreased gradually with egg growth.

Sea cucumbers do not possess a well-developed digestive system for physical chewing or chemical digestion of ingested 

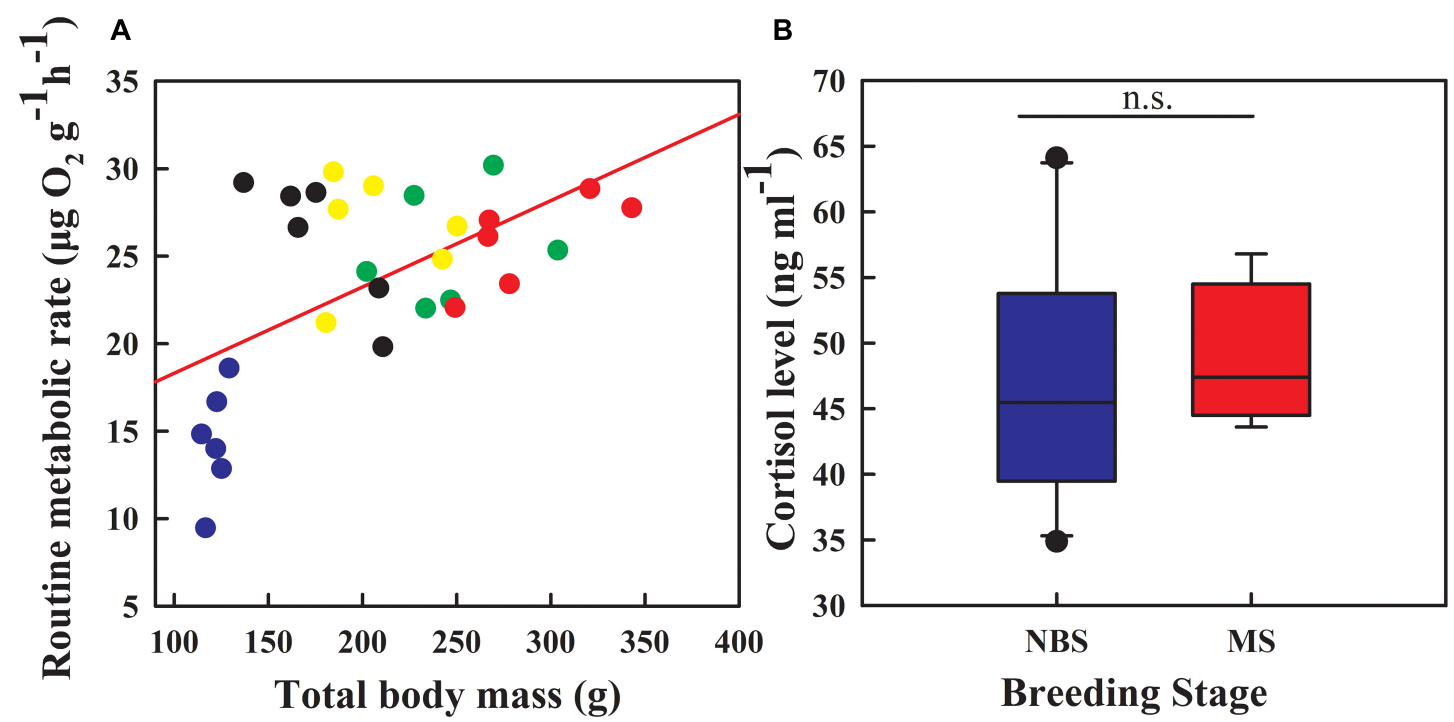

FIGURE 2 | Dynamic changes in routine metabolic rate (A) and cortisol level (B) of sea cucumbers during reproduction. Blue points, day 0; Black points, day 25; Yellow points, day 50; Green points, day 75; Red points, day 100. Blue box plot, non-breeding stage (NBS), day 0; Red box plot, mature stage (MS), day 100. n.s., $P>0.05$.
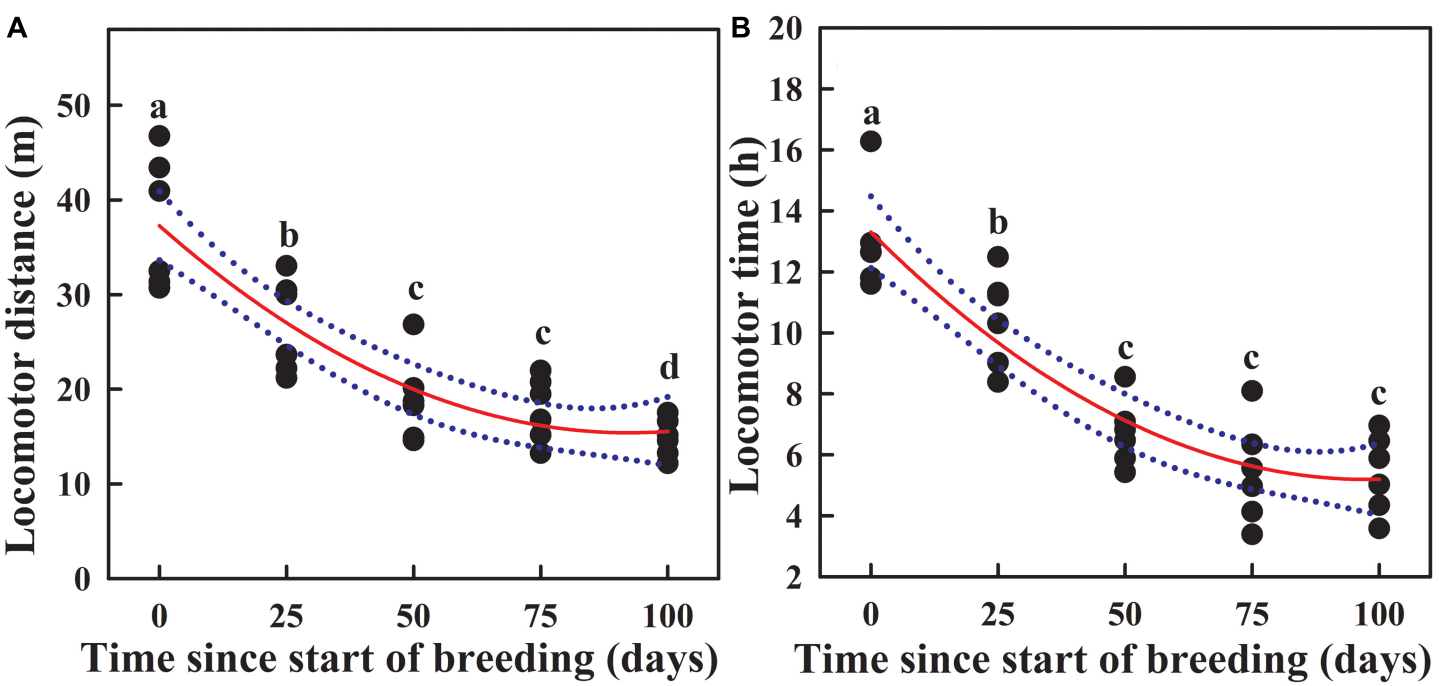

FIGURE 3 | Dynamic changes in locomotor distance (A) and locomotor time (B) of sea cucumbers during reproduction. Dotted lines represent 95\% confidence intervals. Different letters indicate significant differences on different days $(P<0.05)$.

food (Massin, 1982). As a result, most of the ingested energy from food was excreted out of the body as feces (Yuan et al., 2007). However, we showed that sea cucumbers allocate the highest proportion of assimilated energy, an average of $26.48-42.55 \%$, to locomotor function at any reproductive stage. Feeding activity was continuously decreasing over gonad growth. However, both locomotion and reproduction (egg growth and metabolic function associated with reproduction) are energydemanding functions. As a consequence of energy trade-off between reproduction and locomotion, the females gradually reduce unnecessary locomotory activity in order to conserve energy for reproductive success (Fossette et al., 2012). Thus, our results suggest that a decrease in locomotor function in breeding females might serve as a behavioral compensation for the decreased energy acquisition but increased energy demand during reproduction. There is also physiological evidence to support the hypothesis of behavioral energy conservation strategy. The energetic state of longitudinal muscle determines the locomotor activity in sea cucumbers, our recent study has reported that the supply of energy in longitudinal muscles decreases gradually during reproduction (Ru et al., 2017). 

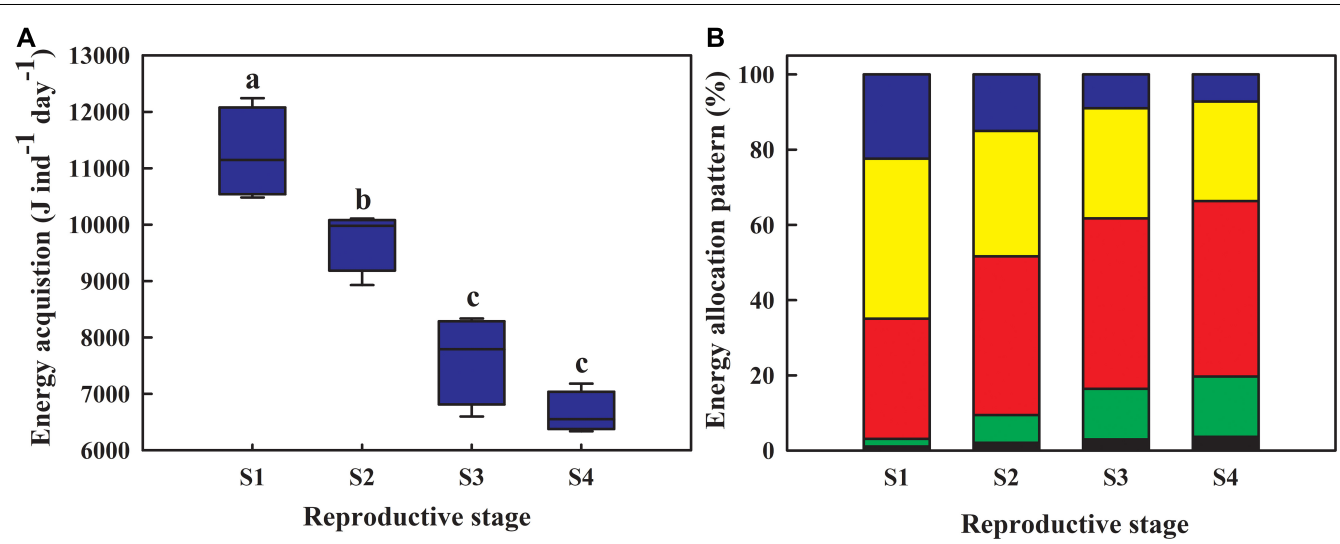

FIGURE 4 | Energy acquisition (A) and allocation patterns (B) of sea cucumbers during reproduction. Blue block indicates the proportion of assimilated energy in somatic tissues; yellow block represents the proportion of energy used in behavioral functions; red block indicates the proportion of energy lost in feces; green block indicates the proportion of energy used in reproduction; black block represents the proportion of energy used in metabolic functions. S1, from day 0 to day 25 during reproduction; S2, from day 25 to day 50; S3, from day 50 to day 75; S4, from day 75 to day 100.

In fact, behavioral plasticity allows animals to adjust their behavioral function to both abiotic and biotic challenges. In particular, plasticity of locomotor behavior is a crucial capacity in sea cucumbers to cope with various challenges on different life history stages. For example, juvenile sea cucumbers can decrease their locomotor activity in order to maintain their survival when faced with strong water flow or intraspecific competition (Pei et al., 2014; Pan et al., 2015). In our study, the results showed that adults could decrease their locomotor activity in order to conserve energy for reproductive success and maintaining energy homeostasis. Similar results also reported in sea turtles. For example, juvenile sea turtles actively decrease their locomotor activity when they are satiated in order to conserve energy for somatic growth (Okuyama et al., 2013). In adults, in order to conserve energy for egg development, turtles also adjust their locomotor activity at low levels to conserve energy for maximum reproductive output (Fossette et al., 2012).

Lastly, this study showed high behavioral (i.e., locomotor activity) and physiological (i.e., routine metabolic rate) variations among individuals in the same environment during reproduction. As a result, sea cucumber gonad mass also showed individual variations within the population. Thus, these results indicate that behavioral and physiological polymorphisms might be the basis for differences of individual reproductive outputs in sea cucumbers. Functionally, the behavioral and physiological polymorphisms in marine invertebrates play an important role in dealing with the uncertainty of the complex marine environment (Dong and Zhang, 2016; Dong et al., 2017). Therefore, the significance of these polymorphisms in maternal traits of A. japonicus requires future studies.

\section{CONCLUSION}

In conclusion, we found that reproduction caused a significant increase in the energy demand in female sea cucumbers. However, the appetite of sea cucumber showed a downward trend over the course of gonad growth. Interestingly, the plasticity of locomotor behavior allows sea cucumbers to decrease locomotor activity in order to conserve energy, thereby accommodating for the increasing energy demand and maintaining energy homeostasis during reproduction. Our study provides new insights into how sea cucumbers accommodate reproductive demands by adopting a behavioral energy conservation strategy.

\section{DATA AVAILABILITY STATEMENT}

The original contributions presented in the study are included in the article/supplementary material, further inquiries can be directed to the corresponding author/s.

\section{ETHICS STATEMENT}

All of the procedures in this study, including animal collection, animal rearing, and euthanasia, were performed under the Guidelines of Ethical Regulations of Animal Welfare of the IOCAS (IOCAS 2013.3).

\section{AUTHOR CONTRIBUTIONS}

XR performed the research. LZ and HY supervised this study. All authors contributed to the article and approved the submitted version.

\section{FUNDING}

This work was supported by the National Natural Science Foundation of China (41906079 and 41876157), the Scientific Research and Technological Development Program of Fangchenggang (AB20014021), and the International Partners Program of the Chinese Academy of Sciences (133137KYSB20180069). 


\section{REFERENCES}

Baeza, J. A., and Fernández, M. (2002). Active brood care in Cancer setosus (Crustacea: Decapoda): the relationship between female behaviour, embryo oxygen consumption and the cost of brooding. Funct. Ecol. 16, 241-251. doi: 10.1046/j.1365-2435.2002.00616.x

Bao, J., Dong, S. L., Tian, X. L., Wang, F., Gao, Q. F., and Dong, Y. W. (2010). Metabolic rates and biochemical compositions of Apostichopus japonicus (Selenka) tissue during periods of inactivity. J. Ocean. Limnol. 28, 218-223. doi: 10.1007/s00343-010-9016-3

Barclay, R. M. R. (1989). The effect of reproductive condition on the foraging behavior of female hoary bats, Lasiurus cinereus. Behav. Ecol. Sociobiol. 24, 31-37. doi: 10.1007/BF00300115

Boehlert, G. W., Kusakari, M., and Yamada, J. (1991). "Oxygen consumption of gestating female Sebastes schlegeli: estimating the reproductive costs of livebearing," in Rockfishes of the Genus Sebastes: Their Reproduction and Early Life History, eds G. W. Boehlert and J. Yamada (Dordrecht: Springer), 81-90. doi: 10.1007/978-94-011-3792-8_9

Dong, Y. W., and Dong, S. L. (2006). Growth and oxygen consumption of the juvenile sea cucumber Apostichopus japonicus (Selenka) at constant and fluctuating water temperatures. Aquac. Res. 37, 1327-1333. doi: 10.1111/j.13652109.2006.01570.x

Dong, Y. W., Dong, S. L., Tian, X. L., Wang, F., and Zhang, M. Z. (2006). Effects of diel temperature fluctuations on growth, oxygen consumption and proximate body composition in the sea cucumber Apostichopus japonicus Selenka. Aquaculture 255, 514-521. doi: 10.1016/j.aquaculture.2005.12.013

Dong, Y. W., and Zhang, S. (2016). Ecological relevance of energy metabolism: transcriptional responses in energy sensing and expenditure to thermal and osmotic stresses in an intertidal limpet. Funct. Ecol. 30, 1539-1548. doi: 10.1111/ $1365-2435.12625$

Dong, Y. W., Li, X. X., Choi, F. M. P., Williams, G. A., Somero, G. N., and Helmuth, B. (2017). Untangling the roles of microclimate, behaviour and physiological polymorphism in governing vulnerability of intertidal snails to heat stress. Proc. R. Soc. B. 284:20162367. doi: 10.1098/rspb.2016.2367

Duisan, L., Salim, G., Soon, T. K., and Ransangan, J. (2021). Sex ratio, gonad and condition indices of mangrove clam, Polymesoda (Geloina) erosa (Bivalvia: Corbiculidae) in Marudu Bay, Sabah, Malaysia: implication for broodstock selection in artificial breeding program. J. Fish. Environ. 45, 106-119.

Fossette, S., Schofield, G., Lilley, M. K. S., Gleiss, A. C., and Hays, G. C. (2012). Acceleration data reveal the energy management strategy of a marine ectotherm during reproduction. Funct. Ecol. 26, 324-333. doi: 10.1111/j.1365-2435.2011. 01960.x

Hamel, J. F., Sun, J. M., Gianasi, B. L., Montgomery, E. M., Kenchington, E. L., Burel, B., et al. (2019). Active buoyancy adjustment increases dispersal potential in benthic marine animals. J. Anim. Ecol. 88, 820-832. doi: 10.1111/1365-2656. 12943

Huang, Y., Fu, S. J., Cooke, S. J., and Xia, J. G. (2020). Is repeatability of metabolic rate influenced by social separation? A test with a teleost fish. Biol. Lett. 16:20190825. doi: 10.1098/rsbl.2019.0825

James, R. S., and Johnston, I. A. (1998). Influence of spawning on swimming performance and muscle contractile properties in the short-horn sculpin. J. Fish Bio. 53, 485-501. doi: 10.1006/jfbi.1998.0722

Jiang, J., Zhao, Z., Pan, Y., Dong, Y., Gao, S., Jiang, B., et al. (2020). Proteomics reveals the gender differences in humoral immunity and physiological characteristics associated with reproduction in the sea cucumber Apostichopus japonicus. J. Proteomics 217:103687. doi: 10.1016/j.jprot.2020.1 03687

Jiang, J., Zhou, Z., Dong, Y., Gao, S., Sun, H., Chen, Z., et al. (2017). Comparative analysis of immunocompetence between females and males in the sea cucumber Apostichopus japonicus. Fish Shellfish Immunol. 63, 438-443. doi: 10.1016/j.fsi. 2017.02.038

Kadri, S., Metcalfe, N. B., Huntingford, F. A., and Thorpe, J. E. (1995). What controls the onset of anorexia in maturing adult female Atlantic salmon? Funct. Ecol. 9, 790-797. doi: 10.2307/2390254

Kraffe, E., Tremblay, R., Belvin, S., LeCoz, J. R., Marty, Y., and Guderley, H. (2008). Effect of reproduction on escape responses, metabolic rates and muscle mitochondrial properties in the scallop Placopecten magellanicus. Mar. Biol. 156, 25-38. doi: 10.1007/s00227-008-1062-4
Krockenberger, A. K., and Hume, I. D. (2007). A flexible digestive strategy accommodates the nutritional demands of reproduction in a free-living folivore, the Koala (Phascolarctos cinereus). Funct. Ecol. 21, 748-756. doi: 10. 1111/j.1365-2435.2007.01279.x

Liang, M., Dong, S. L., Gao, Q. F., Wang, F., and Tian, X. L. (2010). Individual variation in growth in sea cucumber Apostichopus japonicus (Selenck) housed individually. J. Ocean Univ. China 9, 291-296. doi: 10.1007/s11802-010-1739-4

Liu, S. L., Sun, J. C., Ru, X. S., Hamel, J. F., and Mercier, A. (2015). "Broodstock conditioning and spawning," in The Sea Cucumber Apostichopus japonicus: History, Biology and Aquaculture, eds H. S. Yang, J. F. Hamel, and A. Mercier (Amsterdam: Academic Press), 101-110. doi: 10.1016/b978-0-12-799953-1. 00007-6

Marquet, N., Hubbard, P. C., da Silva, J. P., Afonso, J., and Canário, A. V. M. (2018). Chemicals released by male sea cucumber mediate aggregation and spawning behaviours. Sci. Rep. 8:239. doi: 10.1038/s41598-017-18655-6

Massin, C. (1982). "Food and feeding mechanisms: holothuroidea," in Echinoderm Nutrition, eds M. Jangoux and J. Lawrence (Rotterdam: A.A. Balkema Publishers), 43-55. doi: 10.1201/9781003078920-4

McBride, R. S., Somarakis, S., Fitzhugh, G. R., Albert, A., Yaragina, N. A., Wuenschel, M. J., et al. (2015). Energy acquisition and allocation to egg production in relation to fish reproductive strategies. Fish Fish. 16, 23-57. doi: 10.1111/faf.12043

McLean, J. A., and Speakman, J. R. (2000). Morphological changes during postnatal growth and reproduction in the brown long-eared bat Plecotus auritus: implications for wing loading and predicted flight performance. J. Nat. Hist. 34, 773-791. doi: 10.1080/002229300299417

Miles, D. B., Sinervo, B., and Frankino, W. A. (2000). Reproductive burden, locomotor performance, and the cost of reproduction in free ranging lizards. Evolution 54, 1386-1395. doi: 10.1111/j.0014-3820.2000.tb00570.x

Nie, Y. G., Zhang, Z. J., Raubenheimer, D., Elser, J. J., Wei, W., and Wei, F. W. (2015). Obligate herbivory in an ancestrally carnivorous lineage: the giant panda and bamboo from the perspective of nutritional geometry. Funct. Ecol. 29, 26-34. doi: 10.1111/1365-2435.12302

Okuyama, J., Nakajima, K., Noda, T., Kimura, S., Kamihata, H., Kobayashi, M., et al. (2013). Ethogram of immature green turtles: behavioral strategies for somatic growth in large marine herbivores. PLoS One 8:e65783. doi: 10.1371/ journal.pone. 0065783

Pan, Y., Zhang, L. B., Lin, C. G., Sun, J. C., Kan, R. T., and Yang, H. S. (2015). Influence of flow velocity on motor behavior of sea cucumber Apostichopus japonicus. Physiol. Behav. 144, 52-59. doi: 10.1016/j.physbeh.2015.02. 046

Pang, X., Shao, F., Ding, S. H., Fu, S. J., and Zhang, Y. G. (2020). Interspecific differences and ecological correlations of energy metabolism traits in freshwater fishes. Funct. Ecol. 34, 616-630. doi: 10.1111/1365-2435.13505

Pei, S. R., Dong, S. L., Wang, F., Gao, Q. F., and Tian, X. L. (2014). Effects of stocking density and body physical contact on growth of sea cucumber, Apostichopus japonicus. Aquac. Res. 45, 629-636. doi: 10.1111/are. 12004

Pei, S. R., Dong, S. L., Wang, F., Tian, X. L., and Gao, Q. F. (2012). Effects of density on variation in individual growth and differentiation in endocrine response of Japanese sea cucumber (Apostichopus japonicus Selenka). Aquaculture 356, 398-403. doi: 10.1016/j.aquaculture.2012.04.032

Plot, V., Jenkins, T., Robin, J. P., Fossette, S., and Georges, J. Y. (2013). Leatherback turtles are capital breeders: morphometric and physiological evidence from longitudinal monitoring. Physiol. Biochem. Zool. 86, 385-397. doi: 10.1086/ 671127

Pruitt, J. N., and Troupe, J. E. (2010). The effect of reproductive status and situation on locomotor performance and anti-predator strategies in a funnel-web spider. J. Zool. 281, 39-45. doi: 10.1111/j.1469-7998.2009.00677.x

Reiff, T., Jacobson, J., Cognigni, P., Antonello, Z., Ballesta, E., Tan, K. H., et al. (2015). Endocrine remodelling of the adult intestine sustains reproduction in Drosophila. eLife 4:e06930. doi: 10.7554/eLife.06930

Rodríguez, C., Tapia, L., Kieny, F., and Bustamante, J. (2010). Temporal changes in lesser kestrel (Falco naumanni) diet during the breeding season in southern Spain. J. Raptor Res. 44, 120-128. doi: 10.3356/JRR-09-34.1

Ru, X. S., Zhang, L. B., Liu, S. L., and Yang, H. S. (2017). Reproduction affects locomotor behaviour and muscle physiology in the sea cucumber, Apostichopus japonicus. Anim. Behav. 133, 223-228. doi: 10.1016/j.anbehav.2017.09.024 
Ru, X. S., Zhang, L. B., Liu, S. L., Jiang, Y. N., and Li, L. (2021). Physiological traits of income breeding strategy in the sea cucumber Apostichopus japonicus. Aquaculture 539:736646. doi: 10.1016/j.aquaculture.2021.736646

Ru, X. S., Zhang, L. B., Liu, S. L., Sun, J. C., and Yang, H. S. (2018). Energy budget adjustment of sea cucumber Apostichopus japonicus during breeding period. Aquac. Res. 49, 1657-1663. doi: 10.1111/are.13621

Seigel, R. A., Huggins, M. M., and Ford, N. B. (1987). Reduction in locomotor ability as a cost of reproduction in gravid snakes. Oecologia 73, 481-485. doi: 10.1007/BF00379404

Shine, R. (2003). Locomotor speeds of gravid lizards: placing 'costs of reproduction' within an ecological context. Funct. Ecol. 17, 526-533. doi: 10.1046/j.1365-2435. 2003.00756.x

Sokolov, V., Lecomte, N., Sokolov, A., Rahman, M. L., and Dixon, A. (2014). Site fidelity and home range variation during the breeding season of peregrine falcons (Falco peregrinus) in Yamal. Russia. Polar Biol. 37, 1621-1631. doi: 10.1007/s00300-014-1548-0

Speakman, J. R. (2008). The physiological costs of reproduction in small mammals. Phil. Trans. R. Soc. B 363, 375-398. doi: 10.1098/rstb.2007.2145

Sun, J. M., Zhang, L. B., Pan, Y., Lin, C. G., Wang, F., and Yang, H. S. (2018). Effect of water temperature on diel feeding, locomotion behaviour and digestive physiology in the sea cucumber Apostichopus japonicus. J. Exp. Biol. 221(Pt 9):jeb177451. doi: 10.1242/jeb.177451

Tziouveli, V., and Yokoyama, S. (2017). A comparison of the fatty acid profiles of newly hatched, fed, and starved juveniles of Amphioctopus fangsiao (d'Orbigny 1839). Aquac. Int. 25, 1531-1542. doi: 10.1007/s10499-017-0130-5

Unniappan, S., Orchard, I., and Delgado, M. (2020). Neuroendocrine Control of Energy Homeostasis in Non-Mammalian Vertebrates and Invertebrates. Lausanne: Frontiers Media SA, doi: 10.3389/fendo.2020.00404

Winne, C. T., and Hopkins, W. A. (2006). Influence of sex and reproductive condition on terrestrial and aquatic locomotor performance in the semi-aquatic snake Seminatrix pygaea. Funct. Ecol. 20, 1054-1061. doi: 10.1111/j.1365-2435.2006.01180.x

Xu, X. W., Zhang, Z. H., Guo, H. Y., Qin, J. G., and Zhang, X. M. (2020). Changes in aggressive behavior, cortisol and brain monoamines during the formation of social hierarchy in black rockfish (Sebastes schlegelii). Animals 10:2357. doi: 10.3390/ani10122357
Xu, Y. C., Yang, D. B., and Wang, D. H. (2012). No evidence for a trade-off between reproductive investment and immunity in a rodent. PLoS One 7:e37182. doi: 10.1371/journal.pone.0037182

Yuan, X. T., Shao, S. L., Yang, X. L., Yang, D. Z., Xu, Q. Z., Zong, H. M., et al. (2016). Bioenergetic trade-offs in the sea cucumber Apostichopus japonicus (Echinodermata: Holothuroidea) in response to $\mathrm{CO}_{2}$-driven ocean acidification. Environ. Sci. Pollut. Res. 23, 8453-8461. doi: 10.1007/s11356-0166071-0

Yuan, X. T., Yang, H. S., Wang, L. L., Zhou, Y., Zhang, T., and Liu, Y. (2007). Effects of aestivation on the energy budget of sea cucumber Apostichopus japonicus (Selenka) (Echinodermata: Holothuroidea). Acta Ecol. Sin. 27, 3155-3161. doi: 10.1016/S1872-2032(07)60070-5

Yuan, X. T., Yang, H. S., Zhou, Y., Mao, Y. Z., Zhang, T., and Liu, Y. (2006). Salinity effect on respiration and excretion of sea cucumber Apostichopus japonicus (selenka). Ocean. Limnol. Sin. 37, 348-354. doi: 10.1016/j.aquaculture.2010.04. 026

Zhang, H. Y., Xu, Q., Zhao, Y., and Yang, H. S. (2016). Sea cucumber (Apostichopus japonicus) eukaryotic food source composition determined by $18 \mathrm{~s}$ rDNA barcoding. Mar. Biol. 163:153. doi: 10.1007/s00227-016-2931-x

Conflict of Interest: The authors declare that the research was conducted in the absence of any commercial or financial relationships that could be construed as a potential conflict of interest.

Publisher's Note: All claims expressed in this article are solely those of the authors and do not necessarily represent those of their affiliated organizations, or those of the publisher, the editors and the reviewers. Any product that may be evaluated in this article, or claim that may be made by its manufacturer, is not guaranteed or endorsed by the publisher.

Copyright (c) 2021 Ru, Zhang and Yang. This is an open-access article distributed under the terms of the Creative Commons Attribution License (CC BY). The use, distribution or reproduction in other forums is permitted, provided the original author(s) and the copyright owner(s) are credited and that the original publication in this journal is cited, in accordance with accepted academic practice. No use, distribution or reproduction is permitted which does not comply with these terms. 